\section{The molecular pathogenesis of multiple myeloma}

\author{
Niccolò Bolli, ${ }^{1,2}$ Giovanni Martinelli, ${ }^{3}$ \\ Claudio Cerchione ${ }^{3}$ \\ ${ }^{1}$ Department of Oncology and Hemato- \\ Oncology, University of Milan, Italy; \\ ${ }^{2}$ Hematology Unit, Fondazione IRCCS \\ Ca' Granda Ospedale Maggiore \\ Policlinico, Milan, Italy; ${ }^{3}$ Hematology \\ Unit, Istituto Scientifico Romagnolo per \\ lo Studio e la Cura dei Tumori (IRST) \\ IRCCS, Meldola, Italy
}

\begin{abstract}
Multiple Myeloma (MM) is characterized by uncontrolled proliferation and accumulation of clonal plasma cells within the bone marrow. However, the cell of origin is a B-lymphocyte acquiring aberrant genomic events in the germinal center of a lymph node as off-target events during somatichypermutation and class-switch recombination driven by activation-induced-deaminase. Whether pre-germinal center events are also required for transformation, and which additional events are required for disease progression is still matter of debate. As early treatment in asymptomatic phases is gaining traction in the clinic, a better understanding of the molecular pathogenesis of myeloma progression would allow stratification of patients based on their risk of progression, thus rationalizing efficacy and cost of clinical interventions. In this review, we will discuss the development of MM, from the cell of origin through asymptomatic stages such as monoclonal gammopathy of undetermined significance and smoldering MM, to the development of symptomatic disease. We will explain the genetic heterogeneity of MM, one of the major drivers of disease recurrence. In this context, moreover, we will propose how this knowledge may influence future diagnostic and therapeutic interventions.
\end{abstract}

\section{Introduction}

Random mutagenesis is a frequent and likely ubiquitous phenomenon in replicating tissues, stemming from slight intrinsic infidelity of DNA replication and repair processes, and enzymatic modification of DNA bases. ${ }^{1}$ Additionally, exogenous processes may increase this mutation rate. Rarely, these events will result in creation of a variant conferring a proliferative or survival advantage to the cell. In such case, a small pre-clinical proliferation will be observed. ${ }^{2}$ Further acquisition of additional variants may then dictate evolution from these small clonal proliferations, never recognized in clinical practice, to a clinically evident cancer following natural selection acting on the resulting phenotypic diversity. ${ }^{3}$ The complex multicellular microenvironment, the competition for metabolites, oxygen, growth factors, and the necessity for immune escape ${ }^{4}$ will also dictate which clone is the fittest for growth. Genomic plasticity, conferred by the loss of DNA-repair mechanisms and/or acquisition of a hypermutator phenotype, will certainly facilitate the ability to adapt of the tumor cells.

Plasma cell dyscrasias are frequent hematological malignancies, and are usually regarded to as a more complex disease from a genomic point of view as compared to leukemias and lymphomas. ${ }^{5}$ The most frequent conditions, forming a continuous spectrum that can often be observed over time in the same patient, are clinically categorized as monoclonal gammopathy of unknown significance (MGUS), smoldering multiple myeloma (SMM) and active MM.

The diagnosis of MGUS requires the presence of a serum monoclonal protein of $<3 \mathrm{~g} / \mathrm{dL}$ and $<10 \%$ clonal bone marrow (BM) plasma cells, in the absence of myeloma defining events or amyloidosis. MGUS may progress to the more advanced asymptomatic stage of SMM, defined by a serum monoclonal protein of $\geq 3 \mathrm{~g} / \mathrm{dL}$ or $24 \mathrm{~h}$ urinary monoclonal protein $\geq 500 \mathrm{mg}$, and/or $10-60 \%$ clonal BM plasma cells in the absence of myeloma defining events or amyloidosis. Active MM in turn is diagnosed in presence of clonal BM plasma cells $>10 \%$ and/or a biopsy proven bony or extra- medullary plasmacytoma, and one or more myeloma-defining events: end-organ damage (hypercalcemia, renal failure, anemia, lytic bone lesions), $\geq 60 \%$ bone marrow clonal plasma cells, serum free lightchain (FLC) ratio $\geq 100$ (for kappa) or $<0.01$ (for lambda), and $>1$ focal lesion on MRI. ${ }^{6}$

These categories reflect differences in management to prevent the development of end-organ damage, or its prompt recognition and treatment. ${ }^{6}$ From a genomic point of view, the question then is whether this clinical evolution is paralleled by a similar biological evolution of the neoplastic clone, from initiating lesions to those associated with progression and development of an aggressive disease, and whether this can be exploited clinically.

In this review, we will describe recent advances on the molecular pathogenesis of MM. Cell-intrinsic factors involved in the initiation processes of monoclonal gam-
Corresponding Authors: Claudio Cerchione, Hematology Unit, Istituto Scientifico Romagnolo per lo Studio e la Cura dei Tumori (IRST) IRCCS, Via Piero Maroncelli 40, 47014, Meldola (FC), Italy.

Tel. +39.0543 .739100$

E-mail: claudio.cerchione@irst.emr.it

Niccolò Bolli, Hematology Unit, Fondazione IRCCS Ca' Granda Ospedale Maggiore Policlinico, Milan, Italy; Department of Oncology and Hemato-Oncology, University of Milan, Via Francesco Sforza, 35 Milan, Italy.

Tel. +390255033337

E-mail: niccolo.bolli@unimi.it

Key words: Multiple myeloma, tumor evolution, next-generation sequencing, personalized medicine.

Received for publication: 14 December 2020. Accepted for publication: 15 December 2020.

Conflict of interest: the Authors declare no potential conflict of interest.

This work is licensed under a Creative Commons Attribution-NonCommercial 4.0 International License (CC BY-NC 4.0).

CCopyright: the Author(s), 2020

Licensee PAGEPress, Italy

Hematology Reports 2020; 12:9054

doi:10.4081/hr.2020.9054

mopathies will be described in the context of the normal B-cell development. We will then focus on the different patterns of evolution from asymptomatic to aggressive stages of disease and the impact MM heterogeneity has in this process.

\section{Initiating events}

Asymptomatic clonal expansion of hematopoietic cells are nowadays well recognized at the expense of plasma cells, ${ }^{7}$ having been found decades ago through the identification of a monoclonal antibody in the serum through protein electrophoresis. On the contrary, the presence of an asymptomatic clonal B-lymphocytosis has been identified more recently thanks to flow cytometry. ${ }^{8}$ Stem cells can also show similar instances of asymptomatic clonal expansions, identified through next-generation sequencing (NGS). ${ }^{9,10}$ All these evidences confirm the multi-step nature of cancer evolution. In fact, it is thought that all MMs are preceded by an MGUS stage, even if not clinically evident. ${ }^{11}$

Contrary to lymphoproliferative diseases, plasma cell dyscrasias are not classified based on the cell of origin, since the lat- 
ter - a post-germinal center B-lymphocyteis morphologically different from the neoplastic cell encountered in the bone marrow at the time of diagnosis - a plasma cell.

Events leading to transformation of a naïve $B$ cells upon antigen encounter within the germinal center (GC) of lymph nodes are thought to arise from errors during class-switch-recombination (CSR) and somatic hypermutation (SHM) of the B-cell receptor (BCR). These are two processes aimed at increasing antigen affinity to a peculiar antigen and conferring specific effector functions, catalyzed by the activation-induced-deaminase (AID) enzyme. Through the creation of double strand breaks and mutations, AID activity is at risk of off-target mutations and rearrangements. ${ }^{12}$ It is unknown if the transformed cell would need some priming in the form of pre-existing mutations or genomic lesions permissive to the survival upon this AID off-target activity, but this is subject of intense research. Another question is whether this transformation is favored by a germline predisposition. Indeed, the risk of developing a plasma cell dyscrasia is increased twofold in relatives of $\mathrm{MM}$ patients, ${ }^{13}$ and germline transmission of several risk alleles has been described. ${ }^{14,15}$

The transformed B-cell will then home to the BM and differentiate into a plasma cell, giving rise to the clonal expansion clinically recognized as MGUS. Importantly, crucial to this process is the interaction with the microenviroment. ${ }^{16}$

MGUS therefore displays common genetic features with MM: it carries either recurrent translocations of oncogenes with switch regions of the IGH locus or an hyperdiploid (HD) karyotype. The latter consists in multiple trisomies of odd chromosomes with the exception of chromosomes 13 and $17 .{ }^{17}$ IGH translocations are caused by aberrant CSR promoted by AID, as proved by the fact that the rearrangement hotspot is close to the canonical CSR breakpoints. Furthermore, translocation partner genes show mutations with a signature of AID-induced mutations, consistent with a germinal center origin of the event. ${ }^{18}$ Subsequent to the translocation, Ig enhancers promote the overexpression of the recurrent partner genes, consisting in the known oncogenes CCND1, WHSC1, MAF, $M A F B, C C N D 3$ in $\mathrm{t}(11: 14), \mathrm{t}(4: 14)$, $\mathrm{t}(14: 16), \mathrm{t}(14: 20)$ and $\mathrm{t}(6 ; 14)$ respectively. Being initiating events, IGH translocations are almost always clonal, mutually exclusive with each other and with the HD karyotype. ${ }^{12,19}$ On the contrary, mechanisms leading to the generation of an HD karyotype are less clear. In hyperdiploid acute lymphoblastic leukemia, the HD karyotype is thought to derive from a single abnormal cell cycle duplication. ${ }^{19}$ However, analysis of the activity of mutational processes with a constant mutation rate on trisomic chromosomes showed that the number of pregain and post-gain mutations if often different from chromosome to chromosome. This implies that different trisomies can be acquired in different time windows. ${ }^{20}$ Furthermore, only mutations in HD chromosomes acquired before the gain show an off-target AID signature, while mutations acquired after the gain don't show any sign of AID activity. ${ }^{21}$ This demonstrates a germinal center origin of the trisomy. Last, mechanisms linking trisomy to neoplastic transformation are unclear, but may be linked to the expression of oncogenes within the duplicated chromosomes. ${ }^{22}$

The same analysis of the activity of mutational signatures with a constant activity over time has provided bases to enquire when the transformation happens in the life of the patient. Using serial samples from the same patients, the activity of these mutational processes could be extrapolated back in time, concluding that the transforming event could take already in the second or third decade of life. ${ }^{21}$ Subsequently, decades of clonal proliferation and acquisition of additional events would ensue before the clone becomes clinically evident in the form of MGUS.

\section{Genomic features of MGUS}

In MGUS, differently from MM, clonal BM plasma cells are low to absent, the monoclonal protein in the serum is low, and there are no signs of end-organ damage, active MM or amyloidosis. ${ }^{23}$

IGH translocations and HD are transforming events, however they are not sufficient for MM development. In fact, MGUS may display these abnormalities and remain clinically stable. This is the case for the majority of cases, since MGUS progresses at an average rate of $1 \%$ of cases each year, and this rate does not increase even after decades. ${ }^{24}$ This argues against a model of continuous acquisition of additional lesions to drive progression, and on the contrary suggests that clonal sweeps may be driven by stochastic events. The question is therefore what additional genomic events are required for progression. From a point of view of prevalence, the $\mathrm{t}(11 ; 14)$ is more frequent in MGUS, while all other translocations are more prevalent in MM. On the contrary, del(13) and other copy-number alterations (CNAs) are more prevalent in MM. This suggests a differential propensity towards transformation conferred by karyotypic events that can be assessed by FISH or SNP-arrays. ${ }^{25-27}$ Furthermore, the fact that many CNAs can be found at the subclonal level confirms their acquisition after the PC clone has been established.

NGS has the potential to allow a much deeper analysis of the genome of MGUS, highlighting initiating events beyond recurrent translocations and CNAs. However, single-cell RNAseq studies have clearly highlighted how at this stage there is a large number of contaminating, non-clonal PC which may hamper bulk cell analysis. ${ }^{28}$ However, initial targeted DNA-sequencing studies highlighted recurrent mutations in the myeloma genes $N R A S, B R A F, K R A S$, $D I S 3, E G R 1$ and $L T B$. Mutations were less frequent than in active MM and within each case allelic frequencies were suggestive of a late acquisition of the mutation. ${ }^{27,29}$ Importantly, no mutations have been detected in tumor suppressor genes such as TP53, or in genes involved in DNA repair mechanisms as $A T M$ or ATR usually enriched in more advanced phases of the disease. Indeed, MGUS does not display an early and specific single-nucleotide mutational activity that may explain expansion of the tumor clone. This is very different, for example, from what observed in NPM1mutated acute myeloid leukemia, ${ }^{30-32}$ and is more in line with a slowly evolving disease driven by structural events as seems to be the case for most mature lymphoid neoplasms.

Very recently, through the combination of multi-parametric flow-sorting strategy and low-input whole genome library preparation, the genome of highly purified clonal MGUS PCs has been sequenced. ${ }^{33}$ With the addition of SMM and MM cases, a comprehensive analysis of progressive vs non-progressive asymptomatic cases and their comparison to active MM cases has been possible. Results suggested a quite striking difference between MGUS and SMM cases that remained stable in the long term. Stable asymptomatic conditions displayed very little activity of mutational processes besides the AID-activity responsible for disease initiation, ${ }^{34}$ along with reduced numbers of CNAs. Interesting, while the number of trisomies in HD cases were not significantly different between the various conditions, stable asymptomatic cases showed fewer instances of $\operatorname{chr}(1 \mathrm{q})$ gain or amplifications, del $(6 q)$, gain(8q24) involving the $M Y C$ locus, del(16q) as compared to progressive cases. Finally, structural variants and particularly complex events like chromothripsis and templated insertions ${ }^{20}$ were strikingly enriched in progressive cases, suggesting that in the future a molecular signature may 
prognosticate indolent asymptomatic cases much better than the current clinical and laboratory parameters.

\section{Genomic features of SMM}

SMM carries a higher disease burden than MGUS, as by definition clonal plasma cells in the BM must be $>10 \%$ and $<60 \%$. The rate of progression of SMM is $10 \%$ per year in the first 5 years, then declines to $3 \%$ for the next 5 and to $1 \%$ after ten year from diagnosis. ${ }^{35,36} \mathrm{SMM}$ is therefore quite heterogeneous from a clinical point of view, suggesting its definition includes patients ranging from an actual active MM that does not yet satisfy criteria for diagnosis to others with a biologically indolent form similar to MGUS just with more BM PCs.

MGUS patients do not routinely undergo BM examinations during follow-up, therefore it is unusual to catch an evolution from MGUS to SMM even if this is what it is supposed to happen in all progressing cases. This makes it hard to ascertain events associated with initial progression of this asymptomatic conditions, and most of what we know about SMM comes from cases diagnosed $a b$ initio as such. Furthermore, since SMM itself can be stable for years, our knowledge of its evolution is biased towards more aggressive and more rapidly evolving cases.

From a genomic point of view, SMM appears to carry similar genetic abnormalities to active MM, just at a lower frequency. ${ }^{29,37,38}$ An interesting observation came from the whole-genome analysis of paired samples from ultra-high risk SMM progressing to MM. At baseline, the genomic structure of SMM was similar to MM in terms of driver events. This included translocations, CNAs and gene mutations but particularly complex structural events. Known secondary CNAs such as $\operatorname{del}(13 q)$, $\operatorname{del}(6 q), \operatorname{del}(8 p), \operatorname{del}(16 q)$ and $\operatorname{amp}(1 q)$ were also frequent. Differently from MGUS, the structure of high-risk SMM was therefore very similar to that of MM. ${ }^{39}$ Comparing the genome of paired samples, clonal evolution followed one of two main modalities. Authors described a "static progression model", where the subclonal structure grows unchanged from SMM to MM, and a "spontaneous evolution" model, where the subclonal structure of SMM changes because of the acquisition of one or more subclones and/or loss of others at the time of progression to MM. On average, patients would progress in less than one year in the static model, and at a much slower pace in the spontaneous evolution model. Furthermore, analysis of mutational processes active in each subclone was also particularly revealing. AID activity was preponderant in the ancestral clone of each case, again confirming a germinal center origin of the disease. Subclones evolved later in the disease course and responsible for progression showed instead enriched activity of the APOBEC family of DNA deaminases, an aberrant mutational process active across a variety of cancers ${ }^{40}$, shedding some light onto aberrant genomic processes responsible for the acquisition of additional genomic lesions.

More recent studies on much larger sample cohorts have further expanded these findings, and translated them into information that could be used in clinical practice. In particular, $M Y C$ translocations ${ }^{41}$ or $M Y C$ abnormalities, mutations in MAPK genes or DNA repair genes and the $t(4 ; 14)$ all independently predicted progression to $\mathrm{MM}^{42}$ This evidence makes it tempting to assume that genomics can really help prognostication of SMM by identifying at diagnosis cases that will behave like MGUS, and will rarely progress in years owing to the acquisition of additional genomic events, and cases that are de facto MM and already show all features of an aggressive neoplasms. This also highlights the inadequacy of current prognostic scores, mostly based on the tumor burden of $\mathrm{SMM}^{43}$.

\section{Genomic features of MM}

Genomic studies in MM have much changed the perception of the disease in the last 10 years. Dozens of mutated genes, mutational processes, CNAs and complex structural events have been added to the genomic landscape of what initially seemed to be a disease with few karyotypic events ${ }^{44}$ and gene mutations. Initial enthusiasm for the discovery of actionable mutations such as $B R A F \mathrm{~V} 600 \mathrm{E}^{45}$ has nevertheless been curbed by the evidence that MM at diagnosis is a highly heterogeneous disease, ${ }^{18,46,47}$ so that targeted treatment can trigger rapid subclonal outgrowth outcompeting the main

Table 1. Main genomic features of MGUS (1a), SMM (1b) and MM (1c).

$\begin{array}{ll}\text { Stage of disease } & \text { Genomic features } \\ \text { Stable asymptomatic cases } & \text { Fewer instances of chr(1q) gain or amplifications, del(6q), gain(8q24) involving the MYC locus, del(16q) } \\ & \begin{array}{l}\text { as compared to progressive cases } \\ \text { Progressive cases }\end{array} \\ \text { strikingly enriched }\end{array}$
Table la MGUS

\section{Genomic feature}

MYC abnormalities/translocations,

MAPK or DNA repair genes mutations $t$ (4; 14)

\section{Table 1b SMIM}

\section{Genomic feature}

Mutations in CRBN t (11;14)

High-risk lesions:

bi-allelic events in tumor suppressors,

$\operatorname{amp}(1 q)$,

acquisition of an APOBEC signature

\section{Clinical significance}

Indipendently predict SMM progression to MM

\section{Clinical significance}

Predict IMIDs and PI resistance

Predict targeted treatment (venetoclax) responsiveness

Simultaneously resistance to PIs and IMiDs and worse prognosis 
clone at diagnosis. ${ }^{48}$ At the same time, this wealth of information has failed to improve a biological classification of the disease. This is still based on the main cytogenetic events, confirming these are the initiating events that shape the subsequent trajectory of evolution, providing some constraint on the type of alterations required for progres$\operatorname{sion}^{20}$. HD and IGH-translocated cases still provide the mainstay of classification. Within HD cases, about a third associate with CNAs - mostly del(1p), amp(1q), $\operatorname{del}(13 q), \operatorname{del}(14 q), \operatorname{del}(16 q)$ - and the other two thirds with mutations, with a preponderance of mutations of the RAS family. $\mathrm{T}(4 ; 14)$ cases cluster in two categories, frequently associate with del(13q), and in the first they also associate with CNAs, in the second they show fewer CNAs but mutations in DIS3 and FGFR3. t(11:14) cases also fall in two different categories, one with CCND1 and IRF4 mutation, and one with TP53 bi-allelic inactivation. A seventh category is not characterized by any particular structural event, but by a hypermutated genotype. ${ }^{20}$ This evidence reinforces the notion that gene mutations are late events, whose impact is not strong enough to define a genetic category of the disease. Furthermore, most mutated genes are not even expressed in MM. ${ }^{49,50}$ On the contrary, and similar to solid cancers, structural events - many of which are complex and non-recurrent, yet impacting recurrent driver genes - are the events that drive and define the disease. ${ }^{51}$

However, genomic analysis has revealed several important prognostic correlates, most of which are not captured by the R-ISS ${ }^{52}$. A well-known example is what is somehow improperly referred to as "double-hit" multiple myeloma, i.e. cases with ISS stage 3 and chr(1q)amp, or cases with TP53 bi-allelic inactivation. ${ }^{53}$ The definition is somewhat improper since it has been observed how several combinations of genomic events have prognostic relevance, some showing a clear interaction, others simply highlighting an additive effect. ${ }^{54,55}$ Other events associated with worse prognosis include a high activity of the APOBEC mutational process, ${ }^{56}$ IGL-MYC translocations, ${ }^{57}$ TP53 mutations. ${ }^{58}$ While this list is not at all inclusive, the main examples are cited to stress the point that the use of genomic analysis for MM prognostication is still in its infancy, and a comprehensive analysis will be required over large datasets to understand the independent prognostic role of these and other variables to inform clinical decisions.

Aside from prognostic markers, genomics has also opened the search for predictive markers, i.e. events that correlate with response to a specific treatment or lack thereof. However, no such biomarkers have been found to correlate with response to proteasome inhibitors (PIs) or immunomodulatory drugs (IMiDs), the two most used drug classes in induction. Indeed, mutations in $C R B N$ in IMiD-resistant cases $^{59}$ and in proteasome subunit genes in PI-resistant cases $^{60}$ account for a tiny fraction of cases and are not found -or are found at the subclonal level- in the majority of cases that do not respond to such treatments. ${ }^{50,61}$ Indeed, analysis of cases that are simultaneously resistant to PIs and IMiDs suggested instead that chemoresistance in $\mathrm{MM}$ is achieved through the acquisition of high-risk lesions, such as bi-allelic events in tumor suppressors, $\operatorname{amp}(1 \mathrm{q})$, and acquisition of an APOBEC signature. ${ }^{50}$ The described subclonal heterogeneity is responsible for this dynamic evolution of the tumor through lines of treatment and is especially visible in cases of extra-medullary evolution. ${ }^{18,62}$

\section{Potential clinical applications of genomic technologies in plasma cell dyscrasias}

The recent progress prompted by genomics discoveries in MM raised the question as to whether these merit incorporation into routine clinical practice. The paradigm has been set by myeloid malignancies and particularly AML, where genomics has dramatically impacted classification ${ }^{63}$ and prognostication, ${ }^{64}$ prompting the development of clinical-grade NGS sequencing panels. ${ }^{65-67}$ In MM, the nature of the genome of the disease requires that translocation in IGH regions and copy-number abnormalities are captured along with gene mutations, posing additional hurdles to the design of the panel. While several NGS attempts have been successful at matching or outperforming the accuracy of FISH for the detection of such structural events, ${ }^{68-70}$ the perception still is that NGS is a much complicated technique and the extra-information added to common FISH panels is not going to change the way we make clinical decision soon.

This perception may soon be challenged. Starting from SMM, novel prognostic markers incorporating cytogenetic events have been validated, ${ }^{71}$ accepting the notion that disease biology should be more relevant than disease burden for SMM prognostication. ${ }^{72}$ In newly diagnosed MM, treatment paradigms still follow a "one-size fits all" approach, but the example of venetoclax, a new drug class in relapsed-refractory MM that seems to offer a survival advantage in cases with $\mathrm{t}(11 ; 14)$ but a disadvantage in other subgroups ${ }^{73}$ will open the field for personalized treatment in MM based on patient-specific gene lesions. Furthermore, several trials are exploring a risk-adapted approach, if not a "basket" design where personalized treatment is offered based on each patient's gene mutations. ${ }^{74}$ The most immediate application of NGS to the clinic will nevertheless be that of reliable measurement of minimal-residual disease through the sequencing of the patient-specific B-cell receptor rearrangement. The prognostic value of this technique seems to be extremely high, ${ }^{75}$ and this is likely explained by the fact that this technique may overcome the heterogeneity of other phenotypic and genotypic markers of the tumor clone.

Limits of NGS in MM could be represented by the difficulty of obtaining enough DNA from bone marrow aspirates, and by the spatial heterogeneity of the disease. ${ }^{62}$ In this respect, analysis of circulating tumor cells or cell-free tumor DNA could represent a suitable alternative for longitudinal disease monitoring. While initial approaches in MGUS and SMM have been in part disappointing, ${ }^{76,77} \mathrm{MM}$ at diagnosis seem to offer more circulating cells and cfDNA thus allowing a more informative analysis. ${ }^{78,79}$ Limiting amounts of circulating DNA seem also to limit the analysis of peripheral blood to track disease response to treatment so $\mathrm{far}^{80}$. However, there is little doubt that knowledge banks built on thousands of cases of MM, including genomic and clinical details are highlighting prognostic groups that can't be captured by FISH alone. ${ }^{55,53}$ More such efforts are underway and will likely soon reach a consensus on a reduced set of genomic lesions that may explain most of the risk of MM at diagnosis and may amenable to routine clinical-grade detection. Furthermore, the advent of new drug classes will improve the treatment landscape of MM, but potential benefits may be offset by increased costs and toxicity. This mandates that novel biomarkers are found to rationalize treatment, implying that genomic analysis will become routine clinical practice at diagnosis and at each relapse. ${ }^{81}$

\section{References}

1. Alexandrov LB, Nik-Zainal S, Wedge DC, et al. Signatures of mutational processes in human cancer. Nature 2013; 500(7463):415-421.

2. Martincorena I, Raine KM, Gerstung $\mathrm{M}$, et al. Universal Patterns of Selection 
in Cancer and Somatic Tissues. Cell 2017;171(5):1029-1041.e21.

3. Stratton MR, Campbell PJ, Futreal PA. The cancer genome. Nature 2009; 458(7239):719-724.

4. Hanahan D, Weinberg RA. Hallmarks of Cancer: The Next Generation. Cell 2011;144(5):646-674.

5. Ghobrial IM. Myeloma as a model for the process of metastasis: implications for therapy. Blood 2012;120(1):20-30.

6. Rajkumar SV, Dimopoulos MA, Palumbo A, et al. International Myeloma Working Group updated criteria for the diagnosis of multiple myeloma. Lancet Oncol 2014;15(12): e538-48.

7. Kyle RA. Monoclonal gammopathy of undetermined significance. Natural history in 241 cases. Am J Med 1978;64 (5):814-826.

8. Rawstron AC, Green MJ, Kuzmicki A, et al. Monoclonal B lymphocytes with the characteristics of "indolent"; chronic lymphocytic leukemia are present in $3.5 \%$ of adults with normal blood counts. Blood 2002;100(2): 635-639.

9. Jaiswal S, Fontanillas P, Flannick J, et al. Age-related clonal hematopoiesis associated with adverse outcomes. N Engl J Med 2014;371(26):2488-2498.

10. Genovese G, Kähler AK, Handsaker $\mathrm{RE}$, et al. Clonal hematopoiesis and blood-cancer risk inferred from blood DNA sequence. N Engl J Med 2014; 371(26):2477-2487.

11. Landgren O, Kyle RA, Pfeiffer RM, et al. Monoclonal gammopathy of undetermined significance (MGUS) consistently precedes multiple myeloma: a prospective study. Blood 2009;113(22): 5412-5417.

12. Gonzalez D, van der Burg M, GarcíaSanz R, et al. Immunoglobulin gene rearrangements and the pathogenesis of multiple myeloma. Blood 2007;110(9): 3112-3121.

13. Altieri A, Chen B, Bermejo JL, Castro F, Hemminki K. Familial risks and temporal incidence trends of multiple myeloma. Eur J Cancer 2006;42(11): 1661-1670.

14. Went M, Sud A, Försti A, et al. Identification of multiple risk loci and regulatory mechanisms influencing susceptibility to multiple myeloma. Nature Communications 2018;9(1):3707.

15. Bolli N, Barcella M, Salvi E, et al. Next-generation sequencing of a family with a high penetrance of monoclonal gammopathies for the identification of candidate risk alleles. Cancer 2017;123 (19):3701-3708

16. Tricot G. New insights into role of microenvironment in multiple myelo- ma. Lancet 2000;355(9200):248-250.

17. Fonseca R, Bergsagel PL, Drach J, et al. International Myeloma Working Group molecular classification of multiple myeloma: spotlight review. Leukemia 2009;23(12):2210-2221.

18. Bolli N, Avet-Loiseau H, Wedge DC, et al. Heterogeneity of genomic evolution and mutational profiles in multiple myeloma. Nature Communications 2014;52997.

19. Morgan GJ, Walker BA, Davies FE. The genetic architecture of multiple myeloma. Nat Rev Cancer 2012;12(5): 335-348.

20. Maura F, Bolli N, Angelopoulos N, et al. Genomic landscape and chronological reconstruction of driver events in multiple myeloma. Nature Communications 2019;10(1):3835

21. Rustad EH, Yellapantula V, Leongamornlert D, et al. Timing the initiation of multiple myeloma. Nature Communications 2020;11(1):613-14.

22. Bergsagel PL, Kuehl WM, Zhan F, Sawyer J, Barlogie B, Shaughnessy J. Cyclin D dysregulation: an early and unifying pathogenic event in multiple myeloma. Blood 2005;106(1):296-303.

23. Rajkumar SV. Multiple myeloma: 2016 update on diagnosis, risk-stratification, and management. Am J Hematol 2016;91(7):719-734.

24. Kyle RA, Larson DR, Therneau TM, et al. Long-term follow-up of monoclonal gammopathy of undetermined significance. N Engl J Med 2018;378(3):241249.

25. Avet-Loiseau H, Li JY, Morineau N, et al. Monosomy 13 is associated with the transition of monoclonal gammopathy of undetermined significance to multiple myeloma. Intergroupe Francophone du Myélome. Blood 1999;94(8):25832589.

26. Lopez-Corral L, Sarasquete ME, Beà S, et al. SNP-based mapping arrays reveal high genomic complexity in monoclonal gammopathies, from MGUS to myeloma status. Leukemia 2012; 26(12):2521-2529.

27. Mikulasova A, Smetana J, Wayhelova $\mathrm{M}$, et al. Genomewide profiling of copy-number alteration in monoclonal gammopathy of undetermined significance. Eur J Haematol 2016;97(6):568575.

28. Ledergor G, Weiner A, Zada M, et al. Single cell dissection of plasma cell heterogeneity in symptomatic and asymptomatic myeloma. Nat Med 2018;24 (12):1867-1876.

29. Walker BA, Wardell CP, Melchor L, et al. Intraclonal heterogeneity is a critical early event in the development of myeloma and precedes the development of clinical symptoms. Leukemia 2014; 28(2):384-390.

30. Liso A, Bogliolo A, Freschi V, et al. In human genome, generation of a nuclear export signal through duplication appears unique to nucleophosmin (NPM1) mutations and is restricted to AML. Leukemia 2008;22(6):12851289.

31. Borrow J, Dyer SA, Akiki S, Griffiths MJ. Molecular roulette: nucleophosmin mutations in AML are orchestrated through $\mathrm{N}$-nucleotide addition by TdT. Blood [Epub ahead of print].

32. Borrow J, Dyer SA, Akiki S, Griffiths MJ. Terminal deoxynucleotidyl transferase promotes acute myeloid leukemia by priming FLT3-ITD replication slippage. Blood [Epub ahead of print].

33. Oben B, Froyen G, Maclachlan KH, et al. Whole genome sequencing provides evidence of two biologically and clinically distinct entities of asymptomatic monoclonal gammopathies: progressive versus stable myeloma precursor condition. bioRxiv 2020;2020.11.06.372011.

34. Maura F, Degasperi A, Nadeu F, et al. A practical guide for mutational signature analysis in hematological malignancies. Nature Communications 2019;10(1): 2969.

35. Kyle RA, Remstein ED, Therneau TM, et al. Clinical course and prognosis of smoldering (asymptomatic) multiple myeloma. N Engl J Med 2007;356(25): 2582-2590.

36. Rajkumar SV, Landgren O, Mateos MV. Smoldering multiple myeloma. Blood 2015;125(20):3069-3075.

37. Mailankody S, Kazandjian D, Korde N, et al. Baseline mutational patterns and sustained MRD negativity in patients with high-risk smoldering myeloma. Blood Advances 2017;1(22):19111918.

38. Manzoni M, Marchica V, Storti P, et al. Application of next-generation sequencing for the genomic characterization of patients with smoldering myeloma. Cancers 2020;12(5):1332.

39. Bolli N, Maura F, Minvielle S, et al. Genomic patterns of progression in smoldering multiple myeloma. Nature Communications 2018;9(1):3363.

40. Haradhvala NJ, Huang MN, Tian Ng $\mathrm{AW}$, et al. The repertoire of mutational signatures in human cancer. Nature 2020;578(7793):94-101.

41. Misund K, Keane N, Stein CK, et al. MYC dysregulation in the progression of multiple myeloma. Leukemia 2019; 
1135412-5.

42. Bustoros M, Sklavenitis-Pistofidis R, Park J, et al. Genomic profiling of smoldering multiple myeloma identifies patients at a high risk of disease progression. JCO 2020; JCO.20.00437.

43. Lakshman A, Rajkumar SV, Buadi FK, et al. Risk stratification of smoldering multiple myeloma incorporating revised IMWG diagnostic criteria. Blood Cancer J 2018;8(6):59.

44. Fonseca R, Barlogie B, Bataille R, et al. Genetics and cytogenetics of multiple myeloma: a workshop report. Cancer Res 2004;64(4):1546-1558.

45. Chapman MA, Lawrence MS, Keats JJ, et al. Initial genome sequencing and analysis of multiple myeloma. Nature 2011;471(7339):467-472.

46. Lohr JG, Stojanov P, Carter SL, et al. Widespread genetic heterogeneity in multiple myeloma: implications for targeted therapy. Cancer cell 2014;25 (1):91-101.

47. Melchor L, Brioli A, Wardell CP, et al. Single-cell genetic analysis reveals the composition of initiating clones and phylogenetic patterns of branching and parallel evolution in myeloma. Leukemia 2014;28(8):1705-1715.

48. Raab MS, Lehners $\mathrm{N}, \mathrm{Xu}$ J, et al. Spatially-divergent clonal evolution in multiple myeloma: overcoming resistance to BRAF inhibition. Blood 2016;127(17):2155-2157.

49. Rashid NU, Sperling AS, Bolli N, et al. Differential and limited expression of mutant alleles in multiple myeloma. Blood 2014;124(20):3110-3117.

50. Ziccheddu B, Biancon G, Bagnoli F, et al. Integrative analysis of the genomic and transcriptomic landscape of doublerefractory multiple myeloma. Blood Advances 2020;4(5):830-844.

51. Rustad EH, Yellapantula VD, Glodzik $\mathrm{D}$, et al. Revealing the impact of structural variants in multiple myeloma. Blood Cancer Discov 2020; bloodcandisc.0132.2020.

52. Palumbo A, Avet-Loiseau H, Oliva S, et al. Revised international staging system for multiple myeloma: a report from international myeloma working group. J Clin Oncol 2015;33(26):2863-2869.

53. Walker BA, Mavrommatis K, Wardell $\mathrm{CP}$, et al. A high-risk, Double-Hit, group of newly diagnosed myeloma identified by genomic analysis. Leukemia 2019; 33(1):159-170.

54. Walker BA, Mavrommatis K, Wardell $\mathrm{CP}$, et al. Identification of novel mutational drivers reveals oncogene dependencies in multiple myeloma. Blood 2018;132(6):587-597.
55. Bolli N, Biancon G, Moarii M, et al. Analysis of the genomic landscape of multiple myeloma highlights novel prognostic markers and disease subgroups. Leukemia 2018;32(12):26042616.

56. Maura F, Petljak M, Lionetti M, et al. Biological and prognostic impact of APOBEC-induced mutations in the spectrum of plasma cell dyscrasias and multiple myeloma cell lines. Leukemia 2018;32(4):1044-1048.

57. Barwick BG, Neri P, Bahlis NJ, et al. Multiple myeloma immunoglobulin lambda translocations portend poor prognosis. Nature Communications 2019; 10(1):1-13.

58. D’Agostino M, Zaccaria GM, Ziccheddu B, et al. Early relapse risk in newly diagnosed multiple myeloma patients characterized by next-generation sequencing. Clin Cancer Res 2020; clincanres.0951.2020.

59. Kortüm KM, Mai EK, Hanafiah NH, et al. Targeted sequencing of refractory myeloma reveals a high incidence of mutations in CRBN and Ras pathway genes. Blood 2016;128(9):1226-1233.

60. Barrio S, Stühmer T, Da-Viá M, et al. Spectrum and functional validation of PSMB5 mutations in multiple myeloma. Leukemia 2018;591.

61. Corre J, Cleynen A, Robiou du Pont S, et al. Multiple myeloma clonal evolution in homogeneously treated patients. Leukemia 2018;3641046.

62. Rasche L, Chavan SS, Stephens OW, et al. Spatial genomic heterogeneity in multiple myeloma revealed by multiregion sequencing. Nature Communications 2017;8(1):100.

63. Papaemmanuil E, Gerstung $M$, Bullinger L, et al. Genomic classification and prognosis in acute myeloid leukemia. N Engl J Med 2016;374(23): 2209-2221.

64. Gerstung M, Papaemmanuil E, Martincorena I, et al. Precision oncology for acute myeloid leukemia using a knowledge bank approach. Nat Genet 2017;49(3):332-340.

65. Conte N, Varela I, Grove C, et al. Detailed molecular characterisation of acute myeloid leukaemia with a normal karyotype using targeted DNA capture. Leukemia 2013;27(9):1820-1825.

66. McKerrell T, Moreno T, Ponstingl H, et al. Development and validation of a comprehensive genomic diagnostic tool for myeloid malignancies. Blood 2016; 128(1):e1-9.

67. Bolli N, Manes N, McKerrell T, et al. Characterization of gene mutations and copy number changes in acute myeloid leukemia using a rapid target enrichment protocol. Haematologica 2015; 100(2):214-222.

68. Bolli N, Li Y, Sathiaseelan V, et al. A DNA target-enrichment approach to detect mutations, copy number changes and immunoglobulin translocations in multiple myeloma. Blood Cancer J 2016;6(9):e467.

69. Yellapantula V, Hultcrantz M, Rustad $\mathrm{EH}$, et al. Comprehensive detection of recurring genomic abnormalities: a targeted sequencing approach for multiple myeloma. Blood Cancer J 2019;9(12): 101-9.

70. Höllein A, Twardziok SO, Walter W, et al. The combination of WGS and RNASeq is superior to conventional diagnostic tests in multiple myeloma: Ready for prime time? Cancer Genet 2020;2421524.

71. Mateos M-V, Kumar S, Dimopoulos $\mathrm{MA}$, et al. International Myeloma Working Group risk stratification model for smoldering multiple myeloma (SMM). Blood Cancer J 2020;10(10): 102.

72. Maura F, Bolli N, Rustad EH, Hultcrantz M, Munshi N, Landgren O. Moving from cancer burden to cancer genomics for smoldering myeloma: A review. JAMA Oncol [Epub ahead of print].

73. Kumar SK. Venetoclax or placebo in combination with bortezomib and dexamethasone in patients with relapsed or refractory multiple myeloma (BELLI$\mathrm{NI}$ ): a randomised, double-blind, multicentre, phase 3 trial. Lancet Oncol 2020;13.

74. Pawlyn C, Davies FE. Toward personalized treatment in multiple myeloma based on molecular characteristics. Blood 2019;133(7):660-675.

75. Perrot A, Lauwers-Cances V, Corre J, et al. Minimal residual disease negativity using deep sequencing is a major prognostic factor in multiple myeloma. Blood 2018;132(23):2456-2464.

76. Gerber B, Manzoni M, Spina V, et al. Circulating tumor DNA as a liquid biopsy in plasma cell dyscrasias. Haematologica 2018;103(6):e245-e248.

77. Manzoni M, Pompa A, Fabris S, et al. Limits and applications of genomic analysis of circulating tumor DNA as a liquid biopsy in asymptomatic forms of multiple myeloma. HemaSphere 2020;4 (4):e402.

78. Lohr JG, Kim S, Gould J, et al. Genetic interrogation of circulating multiple myeloma cells at single-cell resolution. Science Translational Medicine 2016; 8(363):363ra147-363ra147. 
79. Manier S, Park J, Capelletti M, et al. Whole-exome sequencing of cell-free DNA and circulating tumor cells in multiple myeloma. Nature Communications 2018;9(1):1691.

80. Mithraprabhu S, Morley R, Khong T, et al. Monitoring tumour burden and therapeutic response through analysis of circulating tumour DNA and extracellular RNA in multiple myeloma patients. Leukemia 2019;33(8):2022-2033.

81. Bolli N, Genuardi E, Ziccheddu B,
Martello M, Oliva S, Terragna C. Nextgeneration sequencing for clinical management of multiple myeloma: ready for prime time? Front Oncol 2020; 101046. 